\title{
Intrauterine Growth Restriction Associated with Hematologic Abnormalities: Probable Manifestations of Placental Mesenchymal Dysplasia
}

\author{
Cristina Martinez-Payo, MD ${ }^{1}$ Rocio Alvarez Bernabeu, MD ${ }^{1}$ Isabel Salas Villar, MD² \\ Enrique Iglesias Goy, MD ${ }^{1}$ \\ 1 Servicio de Obstetricia y Ginecología, Hospital Universitario Puerta \\ Address for correspondence Cristina Martinez-Payo, MD, Servicio de \\ de Hierro Majadahonda, Madrid, Spain \\ 2 Servicio de Anatomía Patológica, Hospital Universitario Puerta de \\ Obstetricia y Ginecología, Hospital Universitario Puerta de Hierro \\ Majadahonda, Calle Manuel de Falla n¹, 28222 Majadahonda, Madrid, \\ Hierro Majadahonda, Madrid, Spain \\ Spain (e-mail: cmartinezpy@gmail.com).
}

Am J Perinatol Rep 2015;5:e85-e88.

\begin{abstract}
Keywords

- placental abnormalities

- placentomegaly

- placental vascular abnormality

- hematologic disorders

- intrauterine growth restriction

Introduction Placental mesenchymal dysplasia is a rare vascular disease associated with intrauterine growth restriction, fetal demise as well as Beckwith-Wiedemann syndrome. Some neonates present hematologic abnormalities possibly related to consumptive coagulopathy and hemolytic anemia in the placental circulation.

Case report We present a case of placental mesenchymal dysplasia in a fetus with intrauterine growth restriction and cerebellar hemorrhagic injury diagnosed in the 20th week of pregnancy. During 26th week, our patient had an intrauterine fetal demise in the context of gestational hypertension. We have detailed the ultrasound findings that made us suspect the presence of hematologic disorders during 20th week.

Discussion We believe that the cerebellar hematoma could be the consequence of thrombocytopenia accompanied by anemia. If hemorrhagic damage during fetal life is found, above all associates with an anomalous placental appearance and with intrauterine growth restriction, PMD should be suspected along other etiologies.
\end{abstract}

Placental mesenchymal dysplasia (PMD) is a rare vascular disease first described by Moscoso et al in $1991 .^{1}$ The differential diagnosis should be with a molar pregnancy because of the similarity of the ultrasound findings: placentomegaly with grape-like vesicles. ${ }^{1}$ Pregnancies with PMD may have an unfavorable evolution. ${ }^{2}$ In some cases there are hematologic abnormalities in the neonates possibly related to consumptive coagulopathy and hemolytic anemia caused by a hypervascular placenta. ${ }^{3-5}$ We present a case of a fetus with intrauterine growth restriction and cerebellar hemorrhagic injury diagnosed in the 20th week of pregnancy. Intrauterine fetal demise occurred in the 26th week. The histological studies performed revealed a placental mesenchymal dysplasia.

received

May 27, 2014

accepted after revision

August 18, 2014

published online

June 9, 2015

\section{Case Report}

A 31-year-old pregnant woman who has had three previous pregnancies: one miscarriage and two full-term pregnancies without any complications. Both newborn babies had a normal weight for their gestational age. In the current pregnancy, the ultrasound findings in the 12th week showed placenta with a normal appearance, nuchal translucency was $1.6 \mathrm{~mm}$, and combined screening revealed a low risk for trisomy $211 / 1,503$ and trisomy $18<1 / 10,000$. Pregnancy-associated plasma protein $A$ and $\beta$-human chorionic gonadotropin ( $\beta$-HCG) levels were 0.3 and 0.9 MoM, respectively.

Copyright $\odot 2015$ by Thieme Medical Publishers, Inc., 333 Seventh Avenue, New York, NY 10001, USA. Tel: +1(212) 584-4662.
License terms

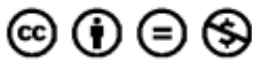

10.1055/s-0034-1394152. ISSN 2157-7005. 


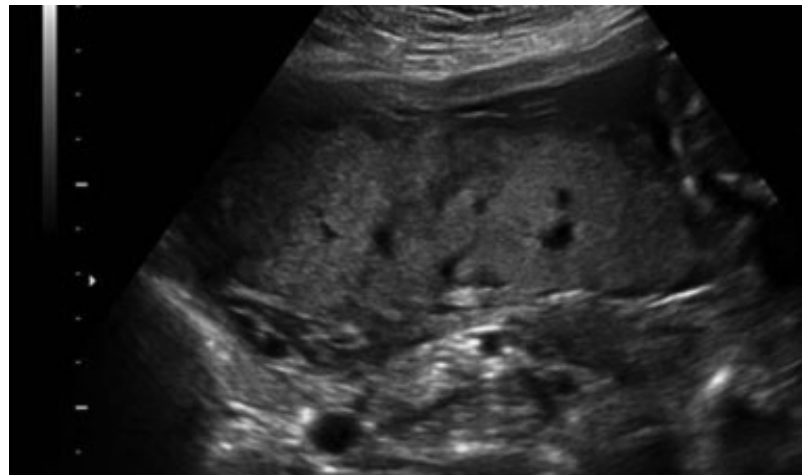

Fig. 1 Placentomegaly with grape-like vesicles.

Doppler ultrasound showed a high uterine artery pulsatility index. On the 3rd day of the 21st week, the ultrasound showed a fetus smaller than the gestational age, the large bones were -3 standard deviation (SD), the cephalic circumference was $-1.7 \mathrm{SD}$, and the abdominal circumference was in the 22nd percentile. Other findings included placentomegaly with hypoechoic areas ( - Fig. 1), severe oligoamnios, a hyperechogenic intestine, and hemorrhagic damage in the left hemisphere of the cerebellum ( - Fig. 2) which was confirmed by magnetic resonance imaging in the 22nd week (-Fig. 3 ). Neither hepatosplenomegaly nor fetal hydrops were detected, although a minor cardiomegaly was diagnosed. The Doppler study revealed normal uterine, umbilical, and ductus venosus flows. Nevertheless, the middle cerebral artery speed was higher than average, $1.5 \mathrm{MoM}$, for the gestational age.

Faced with these findings, an amniocentesis was requested. The karyotype of amniocentesis was 46 XX. Array comparative genomic hybridization analysis revealed no genomic imbalance in the amniocytes. Higher than average concentrations of $\alpha$-protein levels were found in the amniotic fluid. The TORCH (toxoplasmosis, other infections as rubella virus, cytomegalovirus, herpes simplex virus) study revealed no abnormalities.

On the 2nd day of the 24th week of gestation, the amniotic fluid index was decreased, and the growth restriction had

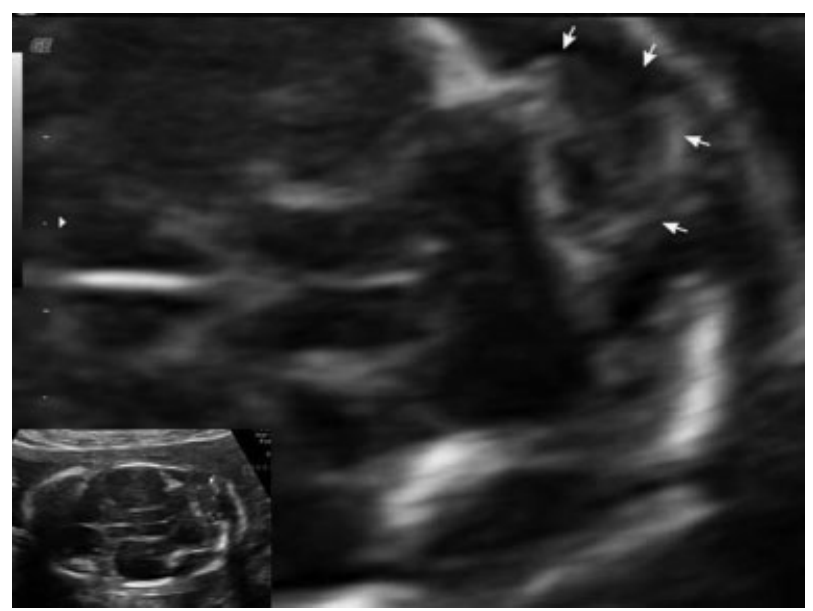

Fig. 2 Cerebellar hematoma (white arrows). The cerebellar hemispheres are asymmetric.

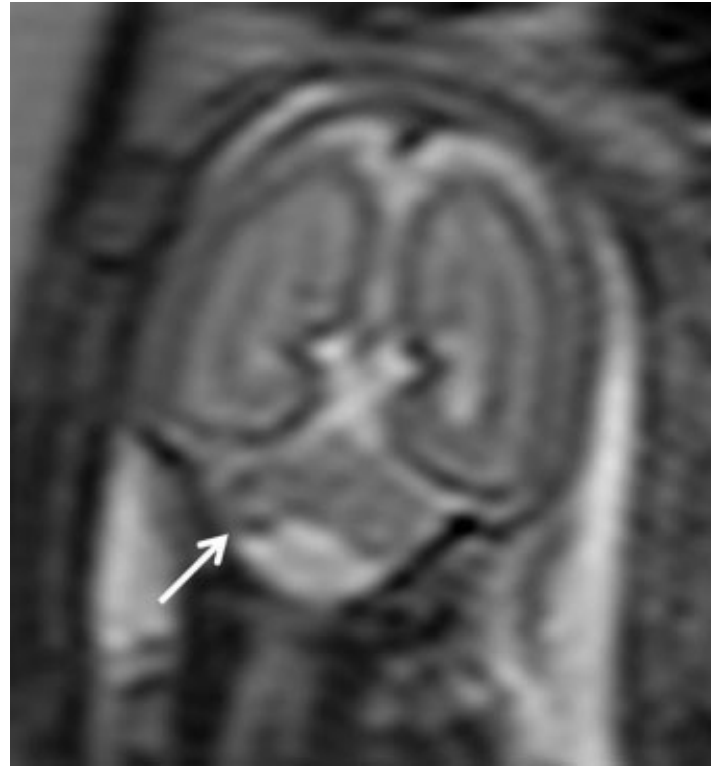

Fig. $3 \mathrm{MRI}$ in the 22nd week. The cerebellar hemorrhage was confirmed (arrow). MRI, magnetic resonance imaging.

become severe with an abdominal circumference under the 5th percentile. The cerebellar hematoma was still present. While the Doppler study showed a normalization of the middle cerebral artery velocity, the umbilical artery pulsatility index increased, but the telediastolic wave was still present. After 3 weeks, our patient came to the emergency room because of abdominal pain and fewer perceived fetal movements. The ultrasound revealed an intrauterine fetal demise. On admission, the patient's arterial pressure was 130/ 83 and the urine analysis showed proteinuria of $7.27 \mathrm{~g}$. Blood tests showed a normal hepatic profile and an hemogram with no coagulation abnormalities. Delivery had to be induced. During the delivery, the blood pressure increased slightly, but never was higher than 150/95. Our patient was hospitalized for 4 days. After the arterial blood pressure and proteinuria were normalized, she was discharged.

The autopsy revealed a $610 \mathrm{~g}$ female newborn, without any external or internal abnormalities, but with a left cerebellar infarct (-Fig. 4). Extreme diffuse extramedullary hematopoiesis was observed in the liver. A tortuous and markedly twisted cord was found with normal vascularization (three vessels). Placental weight was $252 \mathrm{~g}$. The histological study revealed several areas with a focal infarct, abnormal enlarged stem villi surrounded by excessive fibroid material. Trophoblastic hyperplasia was absent. The residual stem villi that were not infarcted were compatible with placental mesenchymal dysplasia.

\section{Discussion}

PMD is a placental vascular abnormality, which can be ultimately diagnosed with a histological study after the delivery. The incidence of this condition is really low $(0.02 \%),{ }^{2}$ even taking into account those that may be misdiagnosed. $^{2}$ The differential rate among female and male fetus is $8: 12 .^{2}$ Ultrasound findings include a thickened 


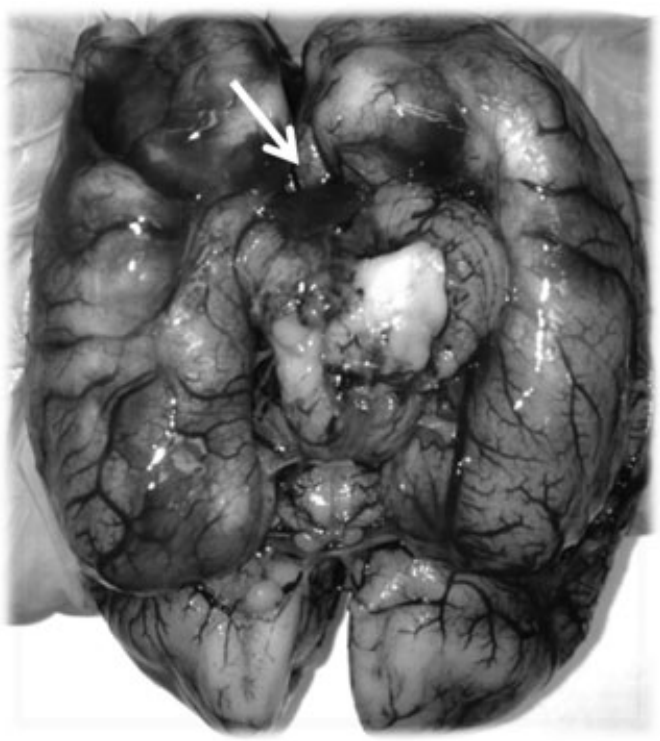

Fig. 4 Image showing fetal brain. Left cerebellar infarct (white arrow). Note the cerebellar asymmetry.

placenta with hypoechoic areas and markedly dilated chorionic vessels. The main differential diagnosis should be with a molar pregnancy. ${ }^{2}$

Fetal abnormalities are found in $38 \%$ of the cases with PMD: $19 \%$ that are suggestive of the Beckwith-Wiedemann syndrome (BWS), intra-abdominal masses, liver cysts, and intrauterine growth restriction (33\%). The outcome of these pregnancies has been often spontaneous preterm labor and the premature rupture of membranes. Maternal clinical manifestations of hypertension during pregnancy are noted in $9 \%$ of the cases. Pregnancy outcomes without complications have been described in only $9 \%$ of the cases. ${ }^{2}$

The levels of HCG and $\alpha$-fetoprotein (AFP) may differ from a normal pregnancy. It is not rare (30\%) to find a lower increase of HCG that could produce an abnormal first-term screening. A high level of AFP in the amniotic fluid is found in $70 \%$ of the cases. A placenta with a molar-like appearance, an elevated concentration of AFP and normal or discreetly elevated HCG is highly suggestive of PMD. ${ }^{6}$

The main histological findings concern the placental stroma. Enlarged abnormal vessels, stromal hyperplasia, and varying degrees of edema are frequently noted, but trophoblastic hyperplasia is invariably not found.

Genetic abnormalities are found in $28 \%$ of these pregnancies: aneuploidy (7\%), BWS and uniparental disomy. ${ }^{6}$ PMD has been associated with an androgenic biparental moisaicim ${ }^{7-10}$ that could explain the frequent association of this condition with BWS. Accordingly, uniparental disomy for imprinted genes located at $11 \mathrm{p} 15.5$ could be the origin of sporadic BWS. ${ }^{11,12}$

Hematologic disorders related to PMD have been described in a few case reports ${ }^{3-5}$ and always in newborn babies. The hypervascularization of the placenta present in PMD, particularly if it coexists with a chorangioma, is related with microangiopathic anemia, thrombocytopenia, and
Kasabach-Merritt coagulopathy phenomenon. In our case, the cerebellar hematoma, placental abnormalities, oligoamnios, and intrauterine growth restriction were diagnosed at the same time. Doppler findings (isolated high speed of middle cerebral artery without other signs of placental insufficiency) could support the hypothesis that the fetus had anemia and probably thrombocytopenia, which has been the cause of cerebellar injury. These hematologic disorders were worsened by a probable hypoxemia, which ultrasound findings showed as oligoamnios and a hyperechogenic intestine. The progressive thrombosis of chorionic vessels that caused the fetal demise in the 26th week of gestation could explain the elevation of umbilical index pulsatility noted during the 24th week.

In conclusion, from our point of view we believe that the cerebellar hematoma could be the consequence of thrombocytopenia accompanied by anemia in a context of PMD. To the best of our knowledge, this is the first case reported in intrauterine life: This association is more frequent in the neonatal period. If hemorrhagic damage during fetal life is found, above all associated with an anomalous placental appearance and with growth restriction, PMD should be suspected, along with other etiologies.

Conflict of Interest

The authors declare no conflict of interest.

\section{References}

1 Moscoso G, Jauniaux E, Hustin J. Placental vascular anomaly with diffuse mesenchymal stem villous hyperplasia. A new clinico-pathological entity? Pathol Res Pract 1991;187(2-3): 324-328

2 Nayeri UA, West AB, Grossetta Nardini HK, Copel JA, Sfakianaki AK. Systematic review of sonographic findings of placental mesenchymal dysplasia and subsequent pregnancy outcome. Ultrasound Obstet Gynecol 2013;41(4):366-374

3 Pham T, Steele J, Stayboldt C, Chan L, Benirschke K. Placental mesenchymal dysplasia is associated with high rates of intrauterine growth restriction and fetal demise: A report of 11 new cases and a review of the literature. Am J Clin Pathol 2006; 126(1):67-78

4 Sengers FB, van Lijnschoten G, van der Sluijs-Bens JP, Porath MM, Dijkman KP. Haematological abnormalities in premature babies due to placental mesenchymal dysplasia [in Dutch]. Ned Tijdschr Geneeskd 2010;154:A1040

5 Koga H, Makimura M, Tanaka H, Sumioki H. Placental mesenchymal dysplasia and fetal hematologic disorder. J Pediatr Hematol Oncol 2014;36(6):e389-e391

6 Robertson M, Geerts LT, de Jong G, Wainwright H. Mesenchymal dysplasia in a monochorionic diamniotic twin pregnancy with review of the differential diagnosis of cystic changes in the placenta. J Ultrasound Med 2007;26(5):689-693

7 Kaiser-Rogers KA, McFadden DE, Livasy CA, et al. Androgenetic/ biparental mosaicism causes placental mesenchymal dysplasia. J Med Genet 2006;43(2):187-192

8 Kapur RP, Cole B, Zhang M, Lin J, Fligner CL. Placental mesenchymal dysplasia and fetal renal-hepatic-pancreatic dysplasia: androgenetic-biparental mosaicism and pathogenesis of an autosomal recessive disorder. Pediatr Dev Pathol 2013;16(3): 191-200 
e88 Placental Mesenchymal Dysplasia Martinez-Payo et al.

9 Robinson WP, Lauzon JL, Innes AM, Lim K, Arsovska S, McFadden DE. Origin and outcome of pregnancies affected by androgenetic/ biparental chimerism. Hum Reprod 2007;22(4):1114-1122

10 Armes JE, McGown I, Williams M, et al. The placenta in BeckwithWiedemann syndrome: genotype-phenotype associations, excessive extravillous trophoblast and placental mesenchymal dysplasia. Pathology 2012;44(6):519-527
11 Paradinas FJ, Sebire NJ, Fisher RA, et al. Pseudo-partial moles: placental stem vessel hydrops and the association with BeckwithWiedemann syndrome and complete moles. Histopathology 2001;39(5):447-454

12 Maher ER, Reik W. Beckwith-Wiedemann syndrome: imprinting in clusters revisited. J Clin Invest 2000;105(3):247-252 